\title{
A SUPERPOSITION THEOREM FOR BOUNDED CONTINUOUS FUNCTIONS
}

\author{
STEPHEN DEMKO
}

\begin{abstract}
It is shown that there exist $2 n+1$ real valued, continuous functions $\phi_{0}, \ldots, \phi_{2 n}$ defined on $\mathbf{R}^{n}$ such that every bounded real valued continuous function on $\mathbf{R}^{n}$ is expressible in the form $\sum_{i=0}^{2 n} g \circ \phi_{i}$ for some $g \in C(\mathbf{R})$. Extensions to some unbounded functions are also made.
\end{abstract}

The Kolmogorov superposition theorem states, in the form of Lorentz [4], that there exist $2 n+1$ real valued functions $\phi_{1}, \ldots, \phi_{2 n+1}$ continuous on the closed unit cube, $I^{n}$, in $\mathbf{R}^{n}$ such that for any $f \in C\left(I^{n}\right)$ there is $g \in C(\mathbf{R})$ such that

$$
f(x)=\sum_{i=1}^{2 n+1} g\left(\phi_{i}(x)\right) \text { for all } x \in I^{n} .
$$

In fact one may take $\phi_{i}$ to be of the form $\phi_{i}\left(x_{1}, \ldots, x_{n}\right)=\sum_{p=1}^{n} e^{p} \psi_{i}\left(x_{p}\right)$ where $\psi_{i} \in C(I)$. The various proofs of this result, e.g., [2], [3], [4], all make use of the uniform continuity of $f$. Recently, Doss [1], has proven the following theorem.

THEOREM. There are functions $\psi_{1}, \ldots, \psi_{2 n-1}, \phi_{1}, \ldots, \phi_{2 n+1} \in C\left(\mathbf{R}^{n}\right)$ such that for any $f \in C\left(\mathbf{R}^{n}\right)$ there exist $g, h \in C(\mathbf{R})$ such that

$$
f(x)=\sum_{i=1}^{2 n-1} h\left(\psi_{i}(x)\right)+\sum_{i=1}^{2 n+1} g\left(\phi_{i}(x)\right) \text { for all } x \in \mathbf{R}^{n} .
$$

Our main result is that there is a choice of $\phi_{1}, \ldots, \phi_{2 n+1}$ such that for bounded functions (and some unbounded ones) we may take $h \equiv 0$. It has as an immediate corollary that every $f \in C\left(\mathbf{R}^{n}\right)$ can be represented in terms of $\phi_{1}, \ldots, \phi_{2 n+1}$, the tangent function, and a function of a single variable. In the spirit of Hedberg [2], we obtain the existence of the $\phi_{i}$ 's by means of a category argument. We then show that if $f$ has compact support, then the representing function $g$ must decay exponentially to zero away from the support of $f$. Paracompactness of $\mathbf{R}^{n}$ enables us to complete the proof. The norm used on functions is always the uniform norm on $\mathbf{R}^{n}$ unless stated otherwise.

Received by the editors November 18, 1976.

AMS (MOS) subject classifications (1970). Primary 26A72.

Key words and phrases. Kolmogorov superposition theorem, Baire category theorem.

- American Mathematical Society 1977 
Throughout this paper $n \geqslant 2$ is a fixed but arbitrary integer and for $m \geqslant 0$ we define the following subsets of $\mathbf{R}^{n}: B_{m}=\{x:\|x\| \leqslant m\}, S_{m}=\{x:\|x\|=$ $m\}$, and $R_{m}=\{x: m-1 \leqslant\|x\| \leqslant m\}$; here we use the $l_{\infty}$ norm. We now recall the cubes of Kolmogorov, [3], [4].

Lemma 1. There exist closed, bounded cubes $\left\{c_{i, k}^{q}: 1 \leqslant q \leqslant 2 n+1, k \geqslant 1\right.$, $i \in \mathbf{N}\}$ and strictly decreasing null sequences $\left\{a_{k}\right\}_{k>1},\left\{b_{k}\right\}_{k>1}$ such that

(1) For fixed $k$ each point of $\mathbf{R}^{n}$ is contained in at least $n+1$ of the cubes $\left\{c_{i, k}^{q}: 1 \leqslant q \leqslant 2 n+1, i \in \mathbf{N}\right\}$.

(2) For fixed $k$ and $q$, the cubes $\left\{c_{i, k}^{q}: i \in \mathbf{N}\right\}$ are disjoint.

(3) $\operatorname{diam}\left(c_{i, k}^{q}\right) \leqslant a_{k}$ for all $i, q$.

(4) $\min _{i} H\left(c_{j, k}^{q}, c_{i, k}^{q}\right) \leqslant b_{k}$ for all $j, q$ where $H(A, B)$ denotes the Hausdorff distance between $A$ and $B$.

The next result shows that the families of cubes can be separated by functions with prescribed ranges.

Lemma 2. There are $2 n+1$ functions $\phi_{1}, \ldots, \phi_{2 n+1}$ in $C\left(\mathbf{R}^{n}\right)$ such that

(a) for $1 \leqslant q \leqslant 2 n+1$ and $m \geqslant 1, \phi_{q}\left(R_{m}\right)=[m, m+2]$ and $\phi_{q}\left(S_{m}\right)=[m$ $+1, m+2]$.

(b) for each $N \geqslant 1$, there is a $k \geqslant N$ such that the sets $\phi_{q}\left(c_{i, k}^{q}\right), 1 \leqslant q \leqslant 2 n$ $+1, i \in \mathbf{N}$, are mutually disjoint.

Proof. The set $M=\left\{\left(\psi_{1}, \ldots, \psi_{2 n+1}\right) \in\left[C\left(\mathbf{R}^{n}\right)\right]^{2 n+1}\right.$ : each $\psi_{q}$ satisfies (a) $\}$ is a nonempty complete metric space if it is given the product-compact-open topology. For $k \geqslant 1$ let $\mathcal{O}_{k}=\left\{\left(\psi_{q}\right) \in M\right.$ : the sets $\psi_{q}\left(c_{i, k}^{q}\right), 1 \leqslant q \leqslant 2 n+1$, $i \in \mathbf{N}$ are mutually disjoint $\}$. It can be verified that for each $N, \cup_{k>N} \theta_{k}$ is open and dense in $M$. By the Baire category theorem $\cap_{N \geqslant 1} \cup_{k>N} \theta_{k}$ is nonvoid; this is statement (b). Q.E.D.

Lemma 3. Let $f \in C\left(\mathbf{R}^{n}\right)$ with $\operatorname{supp} f \subseteq \operatorname{int}\left(\cup_{j=0}^{l} R_{m+j}\right)$ and let $n(n+1)^{-1}$ $<\theta<1$. There exists $g \in C(\mathbf{R})$ such that

(a) $\|g\| \leqslant(n+1)^{-1}\|f\|$

(b) $\left|f(x)-\Sigma_{q} g\left(\phi_{q}(x)\right)\right|<\theta\|f\|$, for all $x \in \mathbf{R}^{n}$ and

(c) supp $g \subseteq[m-1, m+l+3]$.

Proof. Following Lorentz [4, p. 173], we let $\varepsilon>0$ be such that $n(n+1)^{-1}$ $+\varepsilon \leqslant \theta$. Let $N$ be so large that if $\|x-y\|<a_{N}$ (cf. Lemma 1), then $|f(x)-f(y)|<\varepsilon\|f\|$. Let $k \geqslant N$ be such that the sets $\phi_{q}\left(c_{i, k}^{q}\right), 1 \leqslant q \leqslant 2 n$ $+1, i \in \mathrm{N}$, are mutually disjoint. We define $g$ to be constant on each set $\phi_{q}\left(c_{i, k}^{q}\right)$ the constant being the value of $(n+1)^{-1} f$ at the center of $c_{i, k}^{q}$. Extending $g$ linearly to all of $\mathbf{R}$ we see that (a) is satisfied. The proof of (b) follows as in [4]. To prove (c) note that $g\left(\phi_{q}\left(c_{i, k}^{q}\right)\right) \neq\{0\}$ only if $c_{i, k}^{q} \cap$ $\left(\cup_{j=0}^{l} R_{m+j}\right)=\varnothing$ and use part (a) of Lemma 2 .

The next result shows that if $f(x)$ has compact support, then it can be represented in the form (*) where the function $g(x)$ decays to zero exponentially as $x \rightarrow \infty$. 
Lemma 4. Let $f \in C\left(\mathbf{R}^{n}\right)$ with supp $f \subseteq \operatorname{int}\left(R_{m} \cup R_{m+1}\right)$ and let $\theta$ be as in Lemma 3. There exists $g \in C(\mathbf{R})$ and a constant $C$ depending on only $\theta$ such that

(a) for all $x \in \mathbf{R}^{n}, f(x)=\Sigma_{q} g\left(\phi_{q}(x)\right)$ and

(b) $\|g\|_{L_{\infty}[k, k+1]} \leqslant C\|f\| \theta^{|m-k|}$ for all $k \geqslant 0$.

Proof. Let $g_{1}$ satisfy the conditions of Lemma 3 and define $f_{1}(x)=f(x)-$ $\sum_{i} g_{1}\left(\phi_{i}(x)\right)$. Note that $\operatorname{supp} f_{1} \subset \cup_{j=-3}^{4} R_{m+j}$. In general, if $f_{k}$ is defined, we let $g_{k+1}$ be such that $\left\|g_{k+1}\right\| \leqslant(n+1)^{-1}\left\|f_{k}\right\|$ and $\left|f_{k}(x)-\sum_{i} g_{k+1}\left(\phi_{i}(x)\right)\right|<$ $\theta\left\|f_{k}\right\|$ and define $f_{k+1}(x)=f_{k}(x)-\sum_{i} g_{k+1}\left(\phi_{i}(x)\right)$. As in [4], we have $\left\|f_{k}\right\| \leqslant$ $\theta^{k}\|f\|$ and $\left\|g_{k+1}\right\| \leqslant \theta^{k}\|f\|$ which yield (a). By Lemma 3 the $g_{k}$ 's can be chosen so that supp $f_{k} \subseteq \cup_{j=-3 k}^{3 k+1} R_{m+j}$ and supp $g_{k+1} \subseteq[m-3 k-1, m+$ $3 k+4] \cap[0, \infty)$. Consequently, on any interval of the form $[0, m-3 k-1]$ or $[m+3 k+4, \infty)$ the norm of $g$ is bounded by $\|f\| \sum_{r=k+1}^{\infty} \theta^{r}$. This implies (b). Q.E.D.

TheOREM 1. Let $f \in C\left(\mathbf{R}^{n}\right)$ be bounded. There exists $g \in C(\mathbf{R})$ such that for all $x \in \mathbf{R}^{n}, f(x)=\sum_{i=1}^{2 n+1} g\left(\phi_{i}(x)\right)$.

PROof. Since $\mathbf{R}^{n}$ is paracompact, we may write $f(x)=\sum_{m=0}^{\infty} f_{m}(x)$ where $\operatorname{supp} f_{m} \subseteq \operatorname{int}\left(R_{m} \cup R_{m+1}\right)$ and $\left\|f_{m}\right\| \leqslant\|f\|_{L_{\infty}\left(B_{m+1}\right)}$. Let $g_{m}$ satisfy the conclusions of Lemma 4 for $f_{m}$. So,

$$
f(x)=\sum_{m=0}^{\infty} \sum_{i=1}^{2 n+1} g_{m}\left(\phi_{i}(x)\right), \quad x \in \mathbf{R}^{n},
$$

and

$$
\left\|g_{m}\right\|_{L_{\infty}[k, k+1]} \leqslant C \theta^{|m-k|}\|f\|_{L_{\infty}\left(B_{m+1}\right)} .
$$

By the Weierstrass $M$-test, $\sum_{m=0}^{\infty} g_{m} \in C[k, k+2]$ for all $k \geqslant 0$. Therefore, $\sum_{m=0}^{\infty} g_{m} \in C(\mathbf{R})$ and $f(x)=\sum_{i=1}^{2 n+1}\left(\sum_{m=0}^{\infty} g_{m}\left(\phi_{i}(x)\right)\right)$. Q.E.D.

Corollary 1. Let $f \in C\left(\mathbf{R}^{n}\right)$ and assume that $\sum_{m=0}^{\infty} \theta^{m}\|f\|_{L_{\infty}\left(B_{m}\right)}$ converges for some $n(n+1)^{-1}<\theta<1$, then there is $g \in C(\mathbf{R})$ such that

$$
f=\sum_{i=1}^{2 n+1} g \circ \phi_{i} \text {. }
$$

Proof. The assumptions insure that the series $\sum_{m=0}^{\infty} g_{m}$ converges uniformly on compact subsets of $\mathbf{R}$.

We have not been able to extend Theorem 1 to all unbounded functions. However, it is possible to represent unbounded functions by nonlinear superpositions of the $\phi_{i}$ 's.

COROllaRY 2. There is a constant $K>0$ such that for $f \in C\left(\mathbf{R}^{n}\right)$, there exists $g \in C(\mathbf{R})$ with $\|g\| \leqslant K$ and $f(x)=\tan \left\{\sum_{i=1}^{2 n+1} g\left(\phi_{i}(x)\right)\right\}$ for all $x \in \mathbf{R}^{n}$.

Proof. Since $f(x)=\tan \{\operatorname{Arctan} f(x)\}$, apply Theorem 1 to Arc $\tan f(x)$.

REMARK. Theorem 1 can be extended to more general spaces than $\mathbf{R}^{\boldsymbol{n}}$. For example, let $\Omega$ be an open connected subset of $\mathbf{R}^{n}$. In the arguments used 
above, replace $R_{m}$ by $\{x \in \Omega: 1 / m \leqslant \operatorname{dist}(x, \partial \Omega) \leqslant 1 /(m-1)\}, S_{m}$ by $\{x \in \Omega: \operatorname{dist}(x, \partial \Omega)=1 / m\}$ and $B_{m}$ by $\{x \in \Omega: 1 / m \leqslant \operatorname{dist}(x, \partial \Omega)\}$.

\section{REFERENCES}

1. R. Doss, $A$ superposition theorem for unbounded continuous functions, preprint.

2. T. Hedberg, The Kolmogorov superposition theorem, Appendix II, Topics in Approximation Theory by H. S. Shapiro, Springer-Verlag, Berlin and New York, 1971.

3. A. N. Kolmogorov, On the representation of continuous functions of many variables by superpositions of continuous functions of one variable and addition, Amer. Math. Soc. Transl. 28 (1963), 55-59.

4. G. G. Lorentz, Approximation of functions, Holt, Rinehart and Winston, New York, 1966.

School of Mathematics, Georgia Institute of Technology, Atlanta, Georgia 30332 